\section{ARQUEOLOGÍA, DINÁMICA NO LINEAL Y DISCURSO HISTÓRICO}

\author{
ARCHAEOLOGY, NONLINEAR \\ DYNAMICS AND HISTORICAL DISCOURSE
}

\author{
JAMES MCGLADE (*)
}

El tiempo es una invención o no es nada.

(Henri Bergson: L'évolution créatrice)

\section{RESUMEN}

Investigaciones recientes sobre la naturaleza de los sistemas no lineales complejos permiten proponer un punto de vista alternativo de la evolución, en el que se enfatiza la necesidad de un cambio radical en la concepción dominante de causalidad. En esta nueva perspectiva, la autoorganización, la dinámica de bifurcaciones y la evolución caótica se convierten en conceptos claves en la evolución de los sistemas socio-naturales. Las consecuencias de estas ideas para el discurso histórico son profundas puesto que implican una reconsideración de la relación entre contingencia y determinismo. En este artículo se propone que la integración de estos conceptos en un nuevo modelo de práctica arqueológica es un desafío esencial para el futuro de la disciplina.

\begin{abstract}
Recent research into the nature of complex, nonlinear systems suggests an alternative view of evolution, one which underlines the need for a radical shift in the dominant conception of causality -one in which self-organization, bifurcation dynamics and chaotic evolution are seen as core concepts in the evolution of social natural systems. The implications of these ideas for historical discourse are profound since they recast the relationship between contingen-
\end{abstract}

(*) Institute of Archaeology. University College London. London WC1H OP.

El artículo fue remitido en su versión final el 13-VIII-99. cy and determinism. It is argued that the integration of these concepts within a new model of archaeological praxis is a primary challenge for the future of the discipline.

Palabras clave: Teoría arqueológica. Dinámica no lineal. Caos. Historia.

Key words: Archaeological theory. Nonlinear dynamics. Chaos. History.

\section{INTRODUCCIÓN}

En arqueología y en las demás ciencias sociales han aparecido durante la última década una serie de investigaciones que algunas voces han considerado "irrelevantes", "pretenciosas", "resultado de la moda postmoderna", y otras "la revolución más importante de la disciplina en los últimos cien años". Sea cual sea la verdad o la pertinencia de estos comentarios, gran parte del discurso arqueológico más reciente se ha visto implicado en el examen de los procesos y premisas en los que se basa su posición como objeto ontológico de investigación y como disciplina histórica. Se ha procedido a reexaminar la diversidad de plataformas metodológicas y teóricas de la arqueología. Este período de autorreflexión ha estado marcado por una continua polémica sobre la verdadera naturaleza y las posibilidades de recuperación del conocimiento arqueológico.

Uno de los aspectos más destacados de la perspectiva postprocesual es un discurso académico que debe mucho al redescubrimiento de la teoría crítica, sobre todo de la obra de Adorno y de otros miembros de la escuela de Frankfurt y su crítica de 
la práctica y la ideología de la ciencia occidental.A partir de esta inspiración, diversos autores han intentado proponer una metodología postpositivista para la arqueología (p.e. Hodder, 1988; Shanks y Tilley, 1987; Barrett, 1987; Thomas, 1991, 1993; Tilley, 1993; Bender 1993). La toma de posiciones enfrentadas, el elitismo y la búsqueda de una ética superior han caracterizado estos debates y, en ocasiones, han implicado un fervor pseudorreligioso en el intento de establecer la superioridad epistemológica de una manera de conocimiento sobre las demás. Lo que está en juego es la posición y la autoridad de las interpretaciones que hacemos del material arqueológico; es decir, qué tipo de "pasado" queremos construir y para quién.

La relación entre teoría y práctica que se plantea en estos debates debe situarse en el contexto general de la teoría crítica contemporánea: la arqueología se sitúa en un discurso global de las ciencias sociales cuya principal preocupación es la búsqueda de salidas del laberinto de perspectivas que se pone en relación con la condición postmoderna. Una importante consecuencia de este discurso "post mortem de la modernidad" ha sido el examen crítico de la historiografía y de los métodos de reconstrucción histórica y, sobre todo, la preocupación por las formas de entender los procesos que estructuran el cambio social, político y económico. Este panorama implica una crisis de confianza y el rechazo de la historia que conocemos, o al menos, de la problemática posición de las metanarrativas, los grandes modelos de civilización. La crisis se presenta con afirmaciones discutibles sobre el "fin de la historia" (Fukuyama, 1992). Estos debates no se limitan al campo de la historiografía y han llegado a dominar el discurso postmodernista en las ciencias sociales incluyendo, en época más reciente, el de la arqueología (p.e. White, 1971; Jameson, 1991; Hodder, 1992; Shanks y Tilley, 1987).

El razonamiento deconstructivo ha promovido la caída o, al menos, el debilitamiento de la posición arrogante y autocomplaciente que había asumido la historia. Se trata de poner en tela de juicio el discurso histórico (y, por tanto, el arqueológico) cuando asume una trayectoria única y confortable que proclama la superioridad del desarrollo desde el punto de vista del mundo occidental. Hayden White (1971) ha señalado que la historia es un discurso narrativo esencialmente imaginado o inventado y que, por tanto, no puede legitimar supuestas verdades fundamentales. Siguiendo a Kant, sugiere que somos libres de crear el tipo de histo- ria que queramos. Por tanto, la única alternativa lógica es proponer una pluralidad de historias que corresponda a la continua serie de construcciones históricas connotadas de las que se nutre nuestra sociedad. Una de esas construcciones es la perpetuación del mito de la historia como una trayectoria coherente única fruto de una dinámica evolutiva lineal.

Nos enfrentamos a la temible imagen que sugiere la frase de Jameson (1991: 34), según el cual nuestra época se caracteriza por "un único síntoma, la pérdida de la historicidad". Este pensamiento plantea un dilema, porque si rechazamos la idea de historia global que propusieron escritores como Engels, Spengler, y Toynbee, ¿qué pondremos en su lugar? ¿Nos enfrentamos al horror vacui del relativismo, a un mundo de interminables "descripciones gruesas"? Quizás la angustia que plantea la frase de Jameson es equívoca: sin duda las grandes narraciones y su modelo implícito de progreso son discutibles, por no decir inútiles, pero aunque es necesario sustituir este modelo de temporalidad lineal, basado en la acumulación de episodios, la escritura de la historia (y la arqueología es una parte central de ese proyecto) es, sin duda, posible. Se trata de replantear el problema y de resituar el discurso en otro escenario que permita proponer un modo alternativo de causalidad para obtener una lectura diferente del Tiempo. El éxito de este proyecto depende de nuestra capacidad de crear un rapprochement entre la naturaleza temporal de los fenómenos sociales y las temporalidades no lineales que son la base de la dinámica estructural que articula los procesos históricos. Ha de plantearse un cambio radical de la concepción dominante en la teoría arqueológica de la causalidad; un cambio en el que se replantee la relación entre contingencia y determinismo para abordar la naturaleza temporal de la dialéctica socio-histórica tanto a escala local como en la dinámica a largo plazo (1). Podemos preguntarnos cuáles serían las consecuencias de esta nueva lectura de tiempo en arqueología y como afectarían a la construccción de la narrativa histórica. A lo largo de este trabajo trataremos de esas cuestiones y del cambio perceptual y filosófico que exigen, no solo para una epistemología arqueológica, sino como base de una ontología revisada.

(1) La relación entre procesos a corto y a largo plazo es una distinción arbitraria en relación a un conjunto determinado de acontecimientos y a la escala de observación de los mismos. 


\section{TIEMPOS ARQUEOLÓGICOS}

\section{Tiempo objetivado}

Nuestra primera tarea es situar estas cuestiones en el contexto general de la representación del tiempo en la práctica arqueológica. La arqueología está dominada por un discurso cronocéntrico, un discurso que se retuerce sobre si mismo en búsqueda de un orden temporal perfecto y coherente. El pasado se forma a partir de una serie de fases temporales concretas que coinciden y explican los diferentes períodos del desarrollo cultural. Esas periodizaciones constituyen la base aparentemente segura sobre la que se construye la escritura de la historia.

El discurso del tiempo en arqueología se ha caracterizado durante muchos años por una posición objetiva y una naturaleza que no presentaba problemas: el tiempo era evidente. Sin embargo, en los últimos años, la influencia gradual de la teoría postmoderna en las ciencias sociales ha debilitado esta posición y una serie de arqueólogos han presentado propuestas para una nueva teoría del tiempo (p.e., Bailey, 1983, 1987; Shanks y Tilley, 1987; McGlade, 1987; Gosden, 1994; McGlade y van der Leeuw, 1997). Una característica clave de esta reorientación es que, a pesar de que estos autores representan perspectivas muy diferentes, su crítica coincide con el punto de vista postmoderno que propone discusiones sobre la naturaleza del tiempo que han tenido un papel esencial en el proyecto postprocesual en arqueología. La experiencia del tiempo, su cara humana, se ha convertido en la clave de las posiciones contrarias a la omnipresencia de un tiempo objetivo y medido, propio de gran parte de la práctica arqueológica. Con todo, la aparición de un nuevo discurso crítico sobre el tiempo en arqueología no ha logrado situar esa crítica en el contexto de la práctica. Porque más allá de un limitado campo teórico desarrollado por un restringido grupo de autores, persiste el punto de vista normativo en gran parte de la investigación arqueológica ( $c f$. Giddens, 1984; Shanks y Tilley, 1987; Gosden, 1994).

En ese esquema, las múltiples periodicidades que constituyen y definen la existencia socio-cultural se eliminan y se reducen a una serie de fechas. De este modo se construye una cronología escalonada donde se ordenan los sucesos del pasado. Las rupturas y discontinuidades del esquema cronológico se consideran simples anomalías, zonas de vacío que con la aparición de nuevos datos podrán rellenarse. Esas construcciones de desarrollo progresivo se ordenan al servicio de "narrativas sin costuras" que proporcionen la clave para la comprensión y, por tanto, para la interpretación del registro arqueológico. Está claro que se supone que sin esas narrativas la arqueología fracasaría y no sería capaz de reconstruir la historia a largo término. Se hundiría, perdida entre la masa incompleta de materiales, y surgiría el espectro del desorden y lo ininteligible.

Esta búsqueda del orden a partir de la eliminación de la diferencia está enraizada en la tradición intelectual occidental, donde la obsesión por la coherencia y la similitud constituye la base de la clasificación: el desorden, la discontinuidad y la diferencia no tienen lugar en ese esquema. Este énfasis en la similitud y la concordancia distorsiona nuestra lectura del registro arqueológico y nos aleja de la innegable diversidad de los datos, al uniformizar su inherente complejidad que se reduce a una narrativa convincente apoyada en las dataciones cronométricas.

La seductora lógica de este tipo de razonamiento arqueológico es peligrosa y perniciosa: peligrosa porque da preferencia a una dimensión del tiempo única, continua y lineal, y perniciosa porque propone un punto de vista falso del comportamiento humano y de la relación del tiempo con la práctica social. Aunque los arqueólogos nos sentimos atraídos por la lógica de las cronologías "absolutas", debemos enfrentarnos al hecho de que los esquemas construidos a partir de series de fechas de C14, pueden ser equívocos. La aparente objetividad de esta forma de datación es limitada, porque los sucesos que se asignan "científicamente" a un mismo tiempo, pueden tener poca relación entre sí. Y de la misma manera, si tenemos dos sucesos que parecen ser totalmente discontinuos, uno puede proceder de procesos que se remontan a un pasado remoto, y el otro pudo originarse en una acción breve, casi instantánea. Su ecuación cronológica oculta más que revela ( $c f$. Kracauer,1966; Starr, 1966). De hecho, las cronologías absolutas que se construyen a partir de dataciones radiocarbónicas alcanzan su valoración científica a partir de tiempos que ignoran o dejan de lado. Los tiempos intrínsecos de la cultura material una vez usada se olvidan en favor de los tiempos "terminales". El "tiempo absoluto" sólo es el tiempo de la decadencia orgánica, es estadístico y probabilístico (González Marcén y Picazo, 1998). 


\section{Cronologías relativas}

El paso del tiempo y el deseo de reconstruir la secuencia de los acontecimientos históricos se plantea usualmente a partir de la construcción de cronologías relativas por medio de esquemas tipológicos, es decir, tipos estilísticos que se convierten en tiempo fosilizado. Además las clasificaciones tipológicas se elaboran seleccionando unos atributos y olvidando otros, lo que implica pérdida de la diversidad intrínseca del material: el sentido cronológico se reduce al servicio de una ciencia reduccionista. Los problemas de estas prácticas son bien conocidos, aunque sigan siendo básicas en el funcionamiento de la disciplina. Por ejemplo, gran parte de la cronología de la Edad del Bronce europea se ha basado en los cambios de estilo de la cerámica, representados por los fósilestipo cerámicos. De esta manera, los criterios estéticos se convierten en marcadores cronológicos que implican episodios temporales diferentes en la trayectoria histórica de la Edad del Bronce. Todavía es más problemático el hecho de que esos esquemas se han usado también para inferir cambios interculturales.

Parece evidente que utilizar una única categoría de artefactos materiales, frecuentemente la cerámica fina, como base de las inferencias cronológicas resulta, como mínimo, problemático. Si escogiéramos la cerámica grosera en vez de la fina, se obtendría un esquema temporal diferente. Y no sólo eso, puesto que la cerámica grosera se usa normalmente en contextos domésticos, como la cocina u otras tareas de la casa, nos enfrentaríamos en este caso a la relación entre cronología y género. Una investigación reciente (2) ha demostrado que cierto tipo de cronologías convencionales se construyen a partir de objetos funerarios masculinos. Usando un ejemplo del Bronce argárico del Sudeste, la autora muestra que cuando se parte de los ajuares femeninos se logra una ordenación cronológica diferente. Esta propuesta demuestra la arbitrariedad con la que se construyen las inferencias culturales, en base a criterios restringidos que no tienen en cuenta la categoría de género. La reducción de la diferencia implicada en esos métodos es una de las consecuencias de las preocupaciones cronocéntricas de la arqueología.

(2) Paloma González Marcén: Cronologia del Grupo Argárico. Ensayo de fasificación radiométrica a partir de la curva de calibración de alta precisión. Tesis doctoral no publicada, Universitat Autónoma de Barcelona, 1992.

\section{Tiempo cronológico versus tiempo cairológico. Tiempos sociales}

La perdurabilidad del modelo normativo de periodización arqueológica testimonia una forma de inercia intelectual más que la defensa continuada de una posición filosófica. Desde sus orígenes en el siglo XIX, la arqueología y las ciencias naturales han estado sometidas a la tiranía de la narrativa histórica y, por esta razón, han desarrollado una relación singular y predecible con los fenómenos temporales. Al igual que otras disciplinas que emergieron en el siglo pasado, en la épocá de la eclosión evolucionista, la arqueología se constituyó como disciplina a partir de una metodología práctica para la interpretación del paso lineal del tiempo. La atracción de los métodos cronoestratigráficos los convirtió en "naturales", una demostración elegante de la ley del progreso. Durante más de un siglo, el objetivo del conocimiento arqueológico se organizó en función de ese único modelo.

La situación persiste porque la teoría de la evolución revolucionó la comprensión de la historia de la tierra, en gran parte a partir de los esquemas geocronológicos de Hutton y Lyell. Esas obras innovadoras reforzaron la idea de progreso que la Ilustración había promovido. A partir de la obra de Comte, Darwin y Marx, la idea de progreso llegó a dominar la vida intelectual de los siglos XIX y XX, asumiendo el papel de Zeitgeist. En ese clima de principios universalistas, la propia noción de civilización se hizo sinónima de progreso, y Herbert Spencer llegó a considerarlo una ley básica del universo.

La selección de esta perspectiva acumulativa de desarrollo del tiempo implicó la renuncia a otra noción temporal: el tiempo cairológico. Los griegos lo consideraban distinto al tiempo de las mediciones cronológicas; el tiempo cairológico se basa en la experiencia, en la duración de las actividades que realizan los seres humanos (Hahn, 1976: 826). Resulta curioso que, a pesar de que los fenómenos cairológicos tienen un importante papel en los procesos de reproducción social, como demuestran numerosos datos etnográficos y antropológicos, ese modelo temporal haya sido largamente ignorado. Así, las primeras propuestas sociológicas que enfatizaban la naturaleza cualitativa del tiempo frente a una concepción meramente cuantitiva (p.e. Durkheim, 1915; Sorokin y Merton, 1937) no han influido en el discurso de la arqueología hasta época relativamente reciente. El énfasis que esos autores

T. P., 56, n. ${ }^{\circ} 2,1999$ 
daban a la rutina cotidiana del tiempo y a su construcción social aparece tan solo en trabajos recientes (ver la revisión de Gosden, 1994). La premisa central de esta perspectiva puede verse en la reflexión de Durkheim sobre la multiplicidad de ritmos temporales que constituyen la vida social y su énfasis en la importancia de la participación del cuerpo humano en el mundo, siguiendo los trabajos de Husserl y Heidegger.

En ese sentido, la fenomenología se ha preocupado de la naturaleza temporal de las acciones humanas y de la construcción del mundo de la vida (Schutz, 1962), destacando la importancia de las experiencias del tiempo y del espacio. Esa preocupación ha tenido un destacado impacto en la arqueología postprocesual. Puede verse ese proceso, por ejemplo, en el intento de Gosden (1994) de rehabilitar la relevancia del tiempo para las prácticas arqueológicas. Siguiendo a Heidegger plantea que es necesario el análisis de la oposición socie$\mathrm{dad} /$ tiempo y, especialmente, entre tiempo "público" y tiempo "privado". Considera que la naturaleza del proceso social se observa en el encuentro entre los tiempos habituales y los tiempos públicos (Gosden, 1994: 190). Así, "El tiempo público surge como un mecanismo para enfrentarse a los problemas del hábito, a partir de los aspectos temporales de las acciones habituales que forman un ciclo de pautas de vida pensadas y no pensadas" (Gosden, 1994: 189).

Pero la complejidad de la estructuración social no puede reducirse a un esquema de opuestos en el que el tiempo público es una manera de enfrentarse al "problema del hábito". Considerar la noción de acción habitual, rutinaria, como una problemática a priori del espacio social es, como mínimo, un planteamiento dudoso. Además la idea de la oposición binaria entre lo público y lo privado es especialmente problemática para el análisis de la división sexual del trabajo y de las temporalidades que rigen los tiempos del trabajos de las mujeres. Tanto Leccardi (1996) como Picazo (1996) han señalado la compleja serie de ritmos temporales que definen las actividades domésticas. Leccardi y otras teóricas feministas han rechazado explícitamente la división institucional del tiempo en dos esferas opuestas porque perpetúa la ecuación de "tiempo público" como dominio masculino centrado en la producción, frente a un tiempo "privado" o "reproductivo" asociado al trabajo de las mujeres.Además el proceso de insertar la oposición producción /reproducción en contextos arqueológicos puede ser totalmente equívoco, puesto que la propia clasificación es un producto de la modernidad.

Por otro lado, Picazo (1996) hace una propuesta útil para discutir esta problemática a partir del concepto de actividades de mantenimiento, es decir, las relacionadas con el cuidado, alimentación y bienestar del grupo social, tareas que normalmente son realizadas en la esfera doméstica por las mujeres. Estas actividades son frecuentemente devaluadas precisamente porque son "domésticas" $y$, por tanto, de orden inferior. Alternativamente, en el análisis marxista han sido relegadas a la esfera del trabajo "no productivo" (Picazo 1996: 60-61). Desde esa perspectiva parece que el "problema del hábito" y su potencial integración en la esfera pública es un pseudoproblema. Si los aspectos temporales de la vida social tienen valor, el único modelo útil parte de su mutua implicación en un contexto que sea explícitamente de género.

Parece claro, a partir de las observaciones de Hubert y Durkheim, que la arqueología debe implicarse más en las estructuras diarias de la experiencia viva, aunque el recorrido del "tiempo subjetivo" por si mismo es insuficiente para llegar a una narrativa convincente a largo plazo. Por tanto, aunque el interés por el tiempo como construcción social ha añadido un nuevo y valioso nivel teórico a la comprensión de los fenómenos temporales, sobre todo porque ha eliminado las premisas de "objetividad", la perspectiva sigue siendo deficiente en relación a (i) el carácter sincrónico, (ii) la concepción normativa del cambio y (iii) la caracterización del largo plazo.

\section{LA HISTORIOGRAFÍA Y EL TIEMPO DE LA NARRACIÓN}

La escuela de los Annales, fundada por Febvre y Bloch en 1929, ha intentado superar el inmovilismo de la perspectiva del tiempo histórico basado en episodios cronológicos. Con su crítica a la historia concebida como una secuencia de acontecimientos al servicio de la cronología y su extremo cuidado por los detalles, promovió un manifiesto para un cambio radical. Un elemento esencial de su propuesta teórica era que, frente a los esquemas históricos normativos basados en la primacía de los grandes hombres o de los sucesos políticos únicos, son más importantes las correspondencias e interacciones entre las estructuras materiales, sociales y mentales. 
La tesis central de Braudel era que el cambio social es consecuencia de la confluencia de las diferentes temporalidades que estructuran la longue durée. Su énfasis en la duración larga hace de su obra una reflexión importante para la arqueología y una serie de investigadores han destacado su interés desde distintas perspectivas (p.e., Hodder, 1987; Bintliff, 1991; Knapp, 1992). El modelo de Braudel era innovador porque se enfrentaba a las dificultades de conceptualización de la trayectoria de la evolución social y subrayaba la necesidad de una nueva lectura de la relación entre estructura y suceso. Con todo, su preocupación con las formas de estructuración de la historia hace que el papel de los sucesos individuales y de la intervención humana tienda a ser secundario y se sitúe en la marginalidad de la historia. Los individuos son meramente la espuma momentánea de las olas de la historia, una idea que Braudel (1953: 721, 1991: 10-11) representó a través de la imagen de moscas del fuego que en una ocasión vio en Brasil: "sus pálidas luces resplandecían, se difumi-naban, brillaban de nuevo, sin atravesar la noche con una luz verdadera. Lo mismo pasa con los sucesos; más allá del resplandor prevalece la oscuridad".

Además, el interés de Braudel por los procesos geohistóricos da mayor importancia a las variables medioambientales, lo que plantea un problema de determinismo incipiente en el centro de la longue durée. Representa un punto de vista de la estructura como entidad monolítica, que cambia lentamente, lo que minimiza el papel de los acontecimientos contingentes en la emergencia de la estructura socio-espacial. Los sucesos parecen ser engullidos por la inexorable marea de la historia. La aparente sofisticación del método de Braudel casi nos lleva a creer que estamos inmersos en una trayectoria no determinista. Pero es una ilusión, porque la estructura narrativa que trata de construir se basa en la primacía de los procesos geohistóricos sobre la intervención humana: inevitablemente nos deja con una estructura causal totalmente determinista en la que "el largo plazo siempre gana al final".

Quizás el rasgo más notable de las propuestas de Braudel es la incapacidad, a pesar de sus intenciones innovadoras, de conseguir un modelo convincente de la larga duración; no es suficiente con decir que las diferentes periodizaciones representan tiempos distintos. Althusser y Balibar (1969) han criticado el carácter "objetivo" del esquema de
Braudel que, de hecho, congela el tiempo en una serie de etapas sincrónicas, dejando de lado el hecho de que los tiempos sociales están inmersos en una "totalidad" social. Así, aunque Braudel planeó el estudio del Mediterráneo como una demostración de que el tiempo se mueve a velocidades diferentes, no logró mostrar cómo esas temporalidades interaccionan en un sentido dinámico. Además, parece que no admite el cambio episódico o abrupto: la continuidad se enfatiza a expensas de la discontinuidad. La primacía que se da a la longue durée nos presenta un punto de vista esencialmente lineal de la historia. No logra explicitar claramente cómo las diferentes temporalidades, corta, media y larga, implican una variedad de distintos ritmos de cambio de los criterios sociales, políticos y económicos: son esos ritmos de cambio con sus pautas de aceleración y desaceleración los que constituyen la historia estructural de las sociedades y los que pueden permitir un modelo de historia como sistema dinámico no lineal.

\section{DE LA CAUSALIDAD LINEAL A LA NO LINEAL}

\section{Introducción}

Hemos analizado tres esquemas causales de construcción de la narración histórica: el primer modelo opta por un nivel micro proponiendo secuencias de eventos individuales en la ordenación de secuencias cronométricas concretas, hasta formar una trayectoria evolutiva acumulativa. El segundo propone una secuencia temporal que no tiene sentido objetivo independiente de la experiencia del observador y el tercer modelo enfatiza el papel de los acontecimientos sociales y minimiza la importancia de la intervención humana en el cambio estructural a largo plazo. Esos modelos parecen ser irreductibles ya que representan los dos lados de un debate entre suceso y estructura, rasgo omnipresente en las discusiones teóricas actuales en arqueología y en las ciencias sociales en general.

Pero existe otra perspectiva de los mismos temas, una perspectiva cuyo principal mérito es que puede ligar los dos lados del debate uniendo el evento y la estructura en una síntesis evolutiva alternativa. Este modelo supera el dominio de la perspectiva lineal en la consideración arqueológica del tiempo.

T. P., 56, n. ${ }^{\circ} 2,1999$ 


\section{Lineariedad frente a no linearidad}

Que vivimos en un mundo no lineal es un hecho indudable; en un mundo postnewtoniano los grandes efectos no son siempre consecuencia de grandes causas. Sin embargo, seguimos aceptando e insistiendo en el predominio de la causalidad lineal y esa aceptación es un rasgo recurrente en gran parte de la actividad intelectual de nuestra sociedad. Desde la perspectiva del final del siglo XX es sorprendente, por no decir incomprensible: este siglo más que ningún otro se ha destacado por su contribución fundamental a la reteorización del tiempo y del espacio. Es decir, por su irreductibilidad a la relatividad de Einstein y a la mecánica cuántica.Además, una de las revoluciones más importantes de las pasadas dos décadas ha sido el desarrollo de la ciencia no lineal que parte de la evidencia de que el comportamiento evolutivo de muchos sistemas naturales: climáticos, geomorfológicos, ecológicos, biológicos y económicos, tiene propiedades que no pueden predecirse y comportamientos caóticos complejos.

Sin embargo, la paradoja es que el modelo dominante de investigación científica que predomina en el discurso académico parte de premisas lineales. En arqueología e historia, por ejemplo, persistimos en proponer interpretaciones y estructuras de significado que se basan en el determinismo causal. De hecho, una de las imágenes más persistentes del pensamiento occidental es la construcción del tiempo en una trayectoria lineal: todos los acontecimientos pasados, presentes y futuros viajan en esa dirección y se supone, implícitamente, que el quehacer científico implica de alguna manera, la predicción del futuro. Desde la perspectiva de nuestra disciplina, por ejemplo, podría parecer que la relación de la arqueología con la historia a largo plazo es evidente; sin embargo, tradicionalmente se ha concebido esa relación de forma muy limitada. Así, la evolución a largo plazo de las estructuras sociales, políticas e ideológicas se encuadra de forma consistente en un modelo que da primacía al desarrollo temporal de la cultura material. En ese modelo el tiempo se relaciona sobre todo con el desarrollo progresivo de las fuerzas económicas productivas.

La filosofía de progreso que subyace a gran parte de la teoría arqueológica y su preocupación por la noción de los orígenes y por los modelos que muestran el desarrollo de la "complejidad" no es tan solo una consecuencia de las ideas del siglo XIX. El libro de Fukuyama (1992) demuestra la persistente preocupación por un modelo progresivo de la historia y confirma su perduración en la conciencia occidental. La continuidad y fuerza de este punto de vista son preocupantes porque oscurecen la complejidad real que subyace en la dinámica histórica. De hecho, la exclusión de modelos alternativos de evolución y causalidad en el discurso académico ha tenido una profunda influencia en la naturaleza de la explicación en las ciencias sociales en general.

\section{El final del antiguo orden}

En las últimas décadas del siglo XIX el antiguo orden espacio-temporal ya estaba en decadencia. Su punto final (aunque no fue reconocido en aquel momento) se originó a partir de las investigaciones de Henri Poincaré sobre la dinámica cualitativa. Las complejas estructuras topológicas (atractores) pensadas por Poincaré no fueron puestas en práctica hasta la revolución de los ordenadores en los años sesenta pero revelaron nueva y sorprendente información sobre la estructura de la complejidad: orden y caos, que durante largo tiempo se había asumido que eran opuestos irreconciliables, están ligados en una relación insospechada anteriormente. Es un mundo paradójico de caos determinista. La emergencia de la probabilidad y de la geometría no euclidiana junto a los descubrimientos de Poincaré sirvieron para difuminar los límites entre arte y ciencia, y se vio que era falsa la dicotomía establecida entre las ciencias llamadas 'objetivas' y las 'subjetivas'. Con todo, es sorprendente que durante gran parte de este siglo esa falsa dicotomía haya sido y siga siendo una y otra vez replanteada.

Por otra parte, las visiones de ese nuevo mundo iban a provocar otro problema: el de la predicción de los sistemas complejos. El significado revolucionario del tema fue confirmado por el descubrimiento de que muchos sistemas físicos, químicos y biológicos poseen la capacidad de autoorganizarse y de generar espontaneamente estructuras. En esos sistemas la estructura evolutiva se da a través de un proceso de transiciones inestables, provocadas por fenómenos de bifurcación. Las bifurcaciones son puntos de inestabilidad en los que el sistema experimenta formas de reestructuración cualitativa. El comportamiento sin equilibrio es una propiedad intrínsica de los sistemas sociales y puede provocar autoorganización y por tanto, un impulso para la reestructuración cualitativa a medida que el siste- 
ma "evoluciona" de un estado al otro. En ese paradigma la evolución se da a partir de una serie de fases de transición entre estados ordenados y desordenados; bifurcaciones sucesivas que generan nuevo orden estructurado.

Sea cual sea el valor de estas propuestas, desde una perspectiva evolutiva son conceptualmente superiores a las soluciones lineales de los esquemas neoevolutivos convencionales. Además, estas ideas ofrecen una metáfora morfogenética que lleva a la descripción e interpretación de las pautas discontinuas del cambio. Lo importante es destacar el funcionamiento de las propiedades esenciales que no están en equilibrio en los sistemas sociales y la identificación de los umbrales que funcionan como precursores de la transformación social ( $c f$. Allen y McGlade, 1987).

\section{La estructura del desorden}

Un atributo fundamental de estas ideas evolutivas es la importancia de los mecanismos de autorreforzamiento (positive feedback), como los ha llamado Arthur (1988a). En líneas generales, procesos como la reproducción, cooperación y competición son puntos de encuentro del individuo y la comunidad y pueden, bajo condiciones específicas, generar comportamientos inestables potencialmente transformadores.

El predominio de interacciones no lineales hace a los sistemas sociales endémicamente no estables y provoca la emergencia de fluctuaciones erráticas, aperiódicas, en el comportamiento del sistema. Estas fluctuaciones irregulares contienen una estructura sutil y difícil de captar conocida en matemáticas como caos. Fue descrita por primera vez en un artículo esencial de Lorenz (1963) donde se demostraba que el comportamiento caótico es una propiedad de los sistemas puramente deterministas. Como resultado de observaciones posteriores de las ciencias físicas, químicas y biológicas, surgió una especie de consenso sobre el papel de la dinámica caótica en la evolución temporal de sistemas tan diversos como los regímenes climáticos globales, las estructuras biofísicas y procesos fisiológicos del tipo de la corriente sanguínea. Sabemos que es probable que trayectorias aperiódicas, caóticas, se encuentren en todos los sistemas, y la investigación se ha centrado en estas interacciones no lineales. Los sistemas dinámicos no tienen un equilibrio inherente sino que se caracterizan por la existencia de equi- librios múltiples y de series de atractores coexistentes a los que está ligado el sistema y entre los cuales puede oscilar.

Desde nuestra perspectiva actual para la comprensión de la relación entre las estructuras de la duración corta y larga (en términos braudelianos), el descubrimiento de la dinámica compleja es de gran importancia evolutiva, puesto que permite identificar una importante causa de comportamiento emergente. El origen de los comportamientos heterogéneos y asimétricos que se observan en el paisaje arqueológico puede que no sea tan intratable como parece; la creación de estructuras espacialmente heterogéneas puede tener orígenes caóticos. Lo que parece claro es que las oscilaciones no periódicas que proceden de la dinámica caótica, en vez de constituir elementos patológicos, pueden tener un papel operativo significativo en la evolución del sistema, sobre todo porque incrementan la diversidad ( $c f$. Conrad, 1986).

\section{La importancia evolutiva de la inestabilidad}

La discontinuidad, un concepto frecuente en los análisis de la estructura social, no ha recibido la importancia que merece. El énfasis en el orden como una verdad intelectual indudable ha modificado su lugar en la descripción dinámica de los sistemas humanos. De hecho, algunos descubrimientos recientes en ciencias naturales refuerzan el papel fundamental de las bifurcaciones y de las fluctuaciones en la evolución temporal de los sistemas no lineales.

El cambio conceptual ligado al análisis de las propiedades discontinuas en los sistemas sociales es atractivo porque implica considerar las estructuras sociales como entidades autoorganizativas en un sistema complejo abierto y, por tanto, convierte en redundantes las actitudes convencionales respecto a la homogeneidad del tiempo y del espacio. Nos movemos de un mundo definido por las propiedades simétricas clásicas, a un mundo donde la posibilidad de desequilibrio de la organización social puede generar inestabilidades que rompan la simetría. La inestabilidad, lejos de ser la aberración de un sistema "estable" es fundamental para la reproducción del orden social. Desde ese punto de vista, se puede ver la evolución a largo plazo de la estructura social como una historia de discontinuidades en el espacio social; es decir, la historia no es una tela cuidadosamente tejida, sino que ha estado

T. P., 56, n. ${ }^{\circ} 2,1999$ 
punteada por cambios de fase como consecuencia de acciones conscientes e inconscientes. Tales discontinuidades son umbrales de cambio, donde el papel de la intervención humana y/o de los comportamientos ideosincráticos asume una importancia vital en la producción y reproducción de las estructuras sociales.

\section{ARQUEOLOGÍA Y DINÁMICA NO LINEAL}

La consecuencia de estos argumentos para la arqueología (y, de hecho, para las ciencias sociales en general) es que han de verse las relaciones entre los seres humanos y el medioambiente como propias de un sistema dinámico no lineal. Es importante destacar la oposición o tensión entre los ritmos temporales de los procesos naturales y los de las estructuras sociales. Las asimetrías provocadas por los conflictos y las contradicciones proporcionan el contexto para transiciones discontinuas a través de las bifurcaciones que generan los cambios del espacio social.

Hasta época reciente, estas ideas han tenido poca resonancia en los trabajos de teoría arqueológica. Diversas investigaciones recientes han intentado demostrar la importancia del paradigma no lineal para la arqueología (p. e. McGlade, 1995b, 1997a; van der Leeuw, 1990; van der Leeuw y McGlade, 1997a,b). Un rasgo importante de esos estudios es que destacan el papel de los procesos autoorganizativos que a escala local pueden provocar variaciones endógenas y, desde éstas, formas de cambio a escala mayor. Quizás la principal lección de la estructuración evolutiva implicada en la acción de procesos no lineales, es que algunos problemas "permanentes" de la explicación arqueológica, como la transición a la agricultura o el colapso de las sociedades complejas, son enteramente explicables en un paradigma no lineal. Otro tema central es la cuestión del papel de fenómenos de generación espontánea de nuevos modos de comportamiento. En gran medida, estos procesos son la base de la diversidad espacial que encontramos en el paisaje arqueológico.

Por otra parte, es importante destacar que los sistemas humanos, a causa de la presencia de estructuras autorreferenciadas o autocatalíticas en las relaciones y la organización social, son intrínsecamente inestables. Puede observarse en la dinámica de la población y en los complejos procesos de intercambio y distribución, donde modelos de simu- lación de la economía de bienes de prestigio han demostrado que cambios temporales pueden producir trayectorias caóticas (McGlade, 1997b). De modo similar, la investigación de la dinámica estructural de explotación de los recursos por agentes competidores, ha permitido a Huberman (1988) señalar que en situaciones donde la decisión se basa en un conocimiento incompleto y se acompaña de retrasos temporales, pueden darse soluciones periódicas o caóticas en la disposición de recursos.

Lo que comparten esas nuevas explicaciones arqueológicas es el deseo de entender la complejidad evolutiva a partir de un modelo de cambio que elimine mecanismos causales simplistas.Además, proponen que la lectura dinámica no lineal de los problemas arqueológicos convencionales puede dar la clave de una explicación coherente de la relación entre suceso y estructura.

\section{COEVOLUCIÓN SOCIO-NATURAL}

\section{Dinámica humana-medioambiental}

Un aspecto fundamental de la relectura del método y de la teoría arqueológicos propuesta por los arqueólogos postprocesuales ha sido el rechazo a analizar el medioambiente. Probablemente este rechazo surgió como una reacción a los excesos determinísticos de las perspectivas economicistas de la prehistoria, como la de la escuela de paleoeconomía de Cambridge y sus seguidores. Pero, como consecuencia de ese rechazo a los conceptos ecológicos en busca de las "estructuras de sentido" que articulan la vida social, se ha relegado la dinámica medioambiental a la posición de epifenómeno. Este planteamiento ha tenido un efecto debilitador en la discusión teórica porque fomenta el desconocimiento de la complejidad espacio-temporal de la dinámica ecológica y coevolutiva que define la interacción socio-natural.

Como consecuencia se ha llegado a un punto de vista supersocializado de la naturaleza. De esa manera, la teoría arqueológica se ha movido de un tipo de reduccionismo a otro: del reduccionismo medioambiental característico de la teoría paleoeconómica a una serie de puntos de vista diametralmente opuestos pero igualmente limitadores propuestos desde perspectivas teóricas sociales que enfatizan el papel del sentido. La deficiente búsqueda de una arqueología científica "objetiva" ha sido reemplazada por un modelo crítico que se preten- 
de, a la vez, anticientífico y relativista. Esta tendencia "idealista" de las ciencias sociales es limitadora porque no puede tratar de las consecuencias ecológicas y sociales de condiciones no reconocidas de las prácticas sociales en relación al mundo natural, $\mathrm{y}$ de sus consecuencias no intencionales ni previstas. Cualquier representación de esquemas culturales (sociales, simbólicos, estructurales) que se distancia de las temporalidades de la experiencia viva, es problemática. Se puede llegar a construir paisajes ficticios, paisajes cuyos únicos puntos de referencia sean redes residuales de estructuras de significado. Además, el reduccionismo inherente de estas perspectivas tiende a hacer incompletas y, en última instancia, erróneas, las interpretaciones de la dinámica socionatural.

En un esfuerzo para obviar la falsa dicotomía representada por las posiciones procesual / postprocesual, recientemente (McGlade,1995a) se ha enfatizado la importancia de una dinámica coevolutiva para la comprensión de la interacción socio-natural. Se busca proponer una ecología humana no funcionalista en la que la intervención humana pueda resituarse en un contexto socionatural que es, a la vez, evolutivo y contingente. Tenemos que desarrollar estrategias apropiadas para la construcción de una ecología dinámica del espacio social visto como un proceso a largo plazo, dinámico: una ecodinámica humana. Este concepto se refiere a la importancia de la dinámica a largo plazo para generar estructuras y contempla la problemática humano-medioambiental en un esquema de referencia explícitamente ecológico y socio-histórico. Se trata de presentar un punto de vista más completo e integrado de la estructuración social humana, rechazando los modelos de desarrollo evolutivo convencionales y enfatizando en su lugar una perspectiva discontinua, no lineal, que reconoce la importancia crucial de las diferentes temporalidades y de la dinámica dependiente de la escala en la emergencia de la estructura social.

\section{Escalas de los tiempos propios de los fenómenos ecodinámicos humanos}

Uno de los temas más importantes que plantea una perspectiva de ecodinámica humana es la centralidad de la dinámica temporal, es decir, el edificio temporal del mundo socionatural articulado por series de temporalidades que podemos definir como tiempos intrínsecos. Lo que decimos es que los sis- temas biológicos, sociales, políticos y tecnológicos en los que se sitúan los seres humanos tienen sistemas temporales propios (Kümmerer, 1996). Hay tiempos que corresponden a las actividades sociales y naturales, tiempos de los movimientos, tiempos reproductivos que van de la reproducción de la célula, a través de los ciclos estacionales de plantas y animales, hasta las escalas temporales mayores (globales) del tiempo glacial. En un cierto sentido, todas las cosas vivas pueden definirse por un cronotipo o tiempo vital (Fraser, 1981, 1987; Bender y Wellbery, 1991).

Esos tiempos intrínsecos y sus correlatos espaciales forman colectivamente una jerarquía espaciotemporal. En relación a la evolución del paisaje, investigaciones recientes han enfatizado el papel de las temporalidades a diferentes escalas en la estructuración de los entornos semiáridos de la Depresión de Vera, en Almería (McGlade, 1995b; Fedoroff and Courty, 1995). La estructura del paisaje debe considerarse producto de la interrelación de un amplio espectro de temporalidades, desde los procesos más lentos, como los movimientos tectónicos $\left(10^{7}\right)$, los ciclos climáticos $\left(10^{5}\right)$, hasta la dinámica de población $\left(10^{2}\right)$ y otros fenómenos a micronivel $\left(10^{1}\right)$. Es importante destacar que estas temporalidades representan diferentes ritmos de cambio; tenemos velocidades lentas, acumulativas, representadas por los movimientos glaciares y tectónicos, y otras como la dinámica del suelo o de la vegetación que tienen un movimiento rápido. La investigación de diferentes sistemas ecológicos demuestra que la discontinuidad, y sus consecuencias catastróficas, pueden ser resultado de la conjunción de variables "rápidas" y "lentas" (Holling, 1986). Esta complejidad se enfatiza más por la superimposición de sistemas humanos sociales, políticos y económicos. Lo que tenemos en última instancia son series de dependencias intertemporales que definen una dinámica recíproca que sitúa lo social en lo natural y lo natural en lo social.

Este énfasis en los tiempos intrínsecos y sus atributos de escala subraya la importancia del estudio de los procesos evolutivos desde una perspectiva que enfatice el papel de los procesos autorreforzadores en la generación de estructuras sociales. Además, no es importante el cambio per se y los arqueólogos debemos considerar las cuestiones de (i) la medida del cambio y quizás, todavía más importante, (ii) el cambio en la velocidad del cambio. Son esos atributos los que definen los sistemas dinámicos. 
Este último punto es particularmente importante en las perspectivas evolutivas convencionales que consideran el cambio en los sistemas sociales y naturales como el producto de fuerzas contingentes. La emergencia de sucesos no predecibles ni planificados en el centro del proceso social se atribuye al azar. Sin embargo, la confianza con que se propone esa idea no es irrebatible y, en última instancia, puede considerarse reduccionista. No es satisfactoria por su atribución de toda la creatividad humana a lo no previsto, fortuito, al azar. La evolución social no es consecuencia tan sólo de sucesos contingentes pero tampoco de la acción continua de procesos deterministas. Lo que planteamos es que la evolución social debe considerarse como una narrativa compuesta, discontinua.

\section{HACIA UNA NARRATIVA ARQUEOLÓGICA ALTERNATIVA: UN MODELO DE "NECESIDAD CONTINGENTE"}

Una de las primeras implicaciones del paradigma evolutivo alternativo que emerge como una consecuencia de la autoorganización es que la estructuración evolutiva de los sistemas socionaturales debe verse como una confluencia de procesos de estructuración basados en la intervención humana y en redes dinámicas no lineales. Ese modelo enfatiza la importancia de las facetas no pensadas, irracionales, estocásticas de las decisiones humanas y su papel en la generación de estructuras en los momentos críticos de la historia de los sucesos sociales.

El tema crucial es la cuestión de la transformación estructural de los sistemas sociales y el papel de la necesidad y del cambio en la generación de un paisaje de posibilidades de bifurcación. En el lenguaje de la dinámica no lineal, la necesidad (en forma de ideología dominante de una organización política y económica) sigue una historia en cuyo desarrollo predominan procesos deterministas, hasta que se llega a un punto de bifurcación. En ese punto, cuando en el sistema social pueden aparecer fluctuaciones estocásticas o impactos no previstos, la contingencia juega un papel cada vez más influyente para llevar el sistema hacia una nueva trayectoria histórica. Podemos considerar la historia como un modelo de "necesidad contingente" (Shermer, 1995: 63), es decir, "una conjunción de sucesos que tienen un determinado curso de acción delimitado por condiciones anteriores". Es decir, una defini- ción del caos: la acción de un comportamiento estocástico en un sistema determinista. Como dice Ian Stewart (1991: 17) si el comportamiento estocástico es "irregular y no tiene leyes" y el comportamiento determinista está "regido por leyes exactas e inquebrantables", el caos puede definirse como "un comportamiento sin leyes gobernado enteramente por la ley". Es esta coyuntura de necesidad y azar lo que constituye la historia del sistema. La necesidad lleva a un sistema histórico en un cierto sentido hasta que llega a un punto de bifurcación. En ese punto la contingencia juega un papel dominante (en las fluctuaciones) en el impulso del sistema hacia un nuevo camino que, a la larga, desarrollará una nueva necesidad, atenuando la importancia de la contingencia hasta que el sistema llegue a su próxima bifurcación (Prigogine y Stengers, 1979). Aunque parece claro que los determinismos históricos forman parte de los sistemas sociales y económicos y ejercen impacto en los individuos y las sociedades, las contingencias actúan a menudo sean cuales sean las fuerzas (necesidades) que las influyen. De este modo reconfiguran las futuras necesidades (Shermer, 1995: 70).

El mundo social y natural que habitamos está lleno de ejemplos de cómo factores esencialmente arbitrarios y no previstos determinan los posteriores modos históricos. Como ha señalado BrianArthur (1988b), la evolución de las tecnologías nos da ejemplos del papel del azar en la creación de nuevas trayectorias evolutivas irreversibles. Por ejemplo, el primer sistema de teclado para máquinas de escribir apareció en un mercado competitivo con el dominio del sistema QWERTY que todavía existe. Su aparición se debió más al azar que a una ventaja tecnológica estricta; de hecho, este sistema era inferior al menos a otro de sus rivales. En otro ejemplo, Arthur (1988b) discute el papel de la dependencia de sentido histórica y apunta a la importancia del papel del azar y de la necesidad en la dirección de la evolución de los conjuntos urbanos. Al explicar la evolución histórica del urbanismo a nivel regional, usa la analogía de la genética, es decir, que el azar actúa para seleccionar las pautas que se estabilizan, aunque las regiones económicamente atractivas tienen una ventaja selectiva intrínseca y, por tanto, una mayor probabilidad de alcanzar predominio. Estos ejemplos citados por Arthur se conforman al "principio de la historia QWERTY" como sucesos que se juntan de forma no prevista y crean resultados históricos inevitables e irreversibles. 


\section{UNA CONCLUSIÓN REFLEXIVA: ARQUEOLOGÍA COMO HISTORIA A LARGo PLAZO}

¿Cuáles son las implicaciones que surgen de la anterior discusión desde el punto de vista de nuestro interés por los fenómenos temporales? En primer lugar, está claro que la existencia de un mundo no lineal presenta para la arqueología una especie de dilema: un tiempo no lineal significa el final de la historia como la hemos conocido, es decir, como una secuencia de desarrollo y como gran narrativa. En pocas palabras, hace obsoletos los modelos basados en la causalidad aristotélica, y relega la certidumbre de la ciencia newtoniana con su reversibilidad al reino de la ciencia ficción. En contraste a las certezas de pensamiento en Newton / Descartes, con su coherencia temporal, nos enfrentamos a un nuevo territorio probabilístico; nos sumergimos en un océano de tiempos inmersos en un universo cuya única realidad es la ausencia de certeza. Paradójicamente, desde este punto de partida, podemos construir un tipo más sofisticado de interpretación arqueológica.

A pesar de las pretensiones de la retórica postprocesual, la arqueología ha experimentado un renacer, una nueva sensibilidad e incluso una nueva apreciación de los fenómenos temporales. Pero la realidad es diferente más allá del sillón del teórico. La práctica arqueológica todavía está inmersa en el mundo euclidiano con sus geometrías confortables, un mundo de medidas seguras, de lógica metrológica impecable, de realidad "objetiva" que el observador puede contemplar confortablemente desde el exterior.

Pero este mundo mítico espacial y temporal es enteramente ficticio. Los paisajes en los que puede inscribirse la certeza lineal del tiempo han sido reemplazados por un espacio menos predecible; usando palabras de Borges, por una trayectoria más contingente, compuesta de 'senderos que se bifurcan'. Es un mundo desprovisto de verdades universales, un mundo en el que domina lo caprichoso y lo provisional y su relación ambigua con los aspectos deterministas, irrevocables de la evolución. Así, el mundo de los absolutos ha sido reemplazado por un mundo definido sólo por el espacio de posibilidad de la acción. En lugar de un paisaje conocible, medible, tenemos una zona de encuentros donde los esquemas intertemporales crean convergencia y divergencia, no según propensiones deterministas o estocásticas, sino según prioridades regidas por la acción de la dinámica no lineal que subyace en los sistemas sociales y naturales.

La historia, en el análisis final, es una pluralidad de tiempos (continuos y discontinuos), un producto de fuerzas contingentes y deterministas. Pero la historia a menudo se escribe para cumplimentar ideales que son sinónimos del establecimiento de las estructuras de poder, para obtener ventajas políticas, por razones racistas o nacionalistas, o simplemente para promover un punto de vista progresivo, de desarrollo de la evolución social y cultural humana. Es la defensa de estas trayectorias frecuentemente espúreas la que debe ser examinada críticamente si queremos descubrir las estructuras funcionales y de sentido reales que subyacen al compromiso social con el paisaje y con el mundo en general. La coevolución socionatural con sus inestabilidades intrínsecas y su dinámica de no equilibrio es la manifestación más clara de que habitamos y siempre lo hemos hecho, un mundo no lineal. La arqueología debe empezar a preocuparse de las implicaciones de esta situación si quiere contribuir a los debates sobre la historia a largo plazo.

Finalmente, resumiremos las implicaciones de esta discusión sobre la estructuración no lineal, y específicamente sus consecuencias para la investigación arqueológica:

1) un modelo revisado de historia. No hay una única línea de historia: hemos de reconceptualizar la historia como una serie de estructuraciones contingentes que son resultado de la interrelación entre sucesos deterministas, estocásticos y caóticos; la historia no es el símbolo modernista de un árbol con ramas, más bien la "historia es un árbol sin tronco";

2) un modelo revisado de cambio estructural (evolución). Los paisajes arqueológicos no son el centro de un comportamiento adaptativo acumulativo o el palimpsesto acumulativo de la metáfora textual, sino el espacio discontinuo donde se negocia la acción social. Podemos hablar de la historia de bifurcaciones del paisaje;

3) un modelo revisado de causalidad: grandes efectos no son necesariamente resultado de grandes causas; sucesos infinitamente pequeños y/o sucesos insignificantes pueden generar transformaciones mayores;

4) un modelo revisado de tiempo: el determinismo teleológico es ficticio; la realidad es una multiplicidad de temporalidades sociales y naturales caracterizada por diferentes velocidades o tiempos intrínsecos, componentes individuales de un siste- 
ma de paisaje mayor que pueden comportarse de modos muy diferentes en distintos intervalos temporales;

5) posibilidad de caminos evolutivos múltiples: la no linealidad enfatiza la noción de que no hay un "pasado", sino interpretaciones culturalmente mediatizadas del pasado y deben considerarse como producto de una historia de bifurcaciones.

A un nivel fundamental, lo que estos puntos enfatizan es el papel de la dinámica no lineal en la estructuración del tiempo y del espacio y, por tanto, de la organización social, que exige la construcción de una nueva ontología arqueológica, en la que la autoorganización, la dinámica de bifurcaciones y la evolución caótica sean conceptos claves en la evolución de los sistemas socionaturales. La integración de estos conceptos en un nuevo modelo de práctica arqueológica es un desafío de gran importancia para el futuro de la disciplina.

\section{BIBLIOGRAFÍA}

Allen, P.M. y McGlade, J.M. (1987): "Evolutionary drive: the effect of microscopic diversity, error- making and noise". Foundations of Physics, 17: 723-38.

Althusser, L. y Balibar, E. (1969): Para leer "El Capital". Siglo Veintiuno Editores. México.

ARRECHI, F.T. (1992): "A critical approach to complexity and self-organization". La Nueva Critica , 1: 7-40.

ARTHUR, W.B. (1988a): "Self-reinforcing mechanisms in economics". En P.W. Anderson, K.J. Arrow y D. Pines (eds.): The Economy as an Evolving Complex System. Addison-Wesley. Redwood City: 99-123.

- (1988b): "Urban systems and historical path dependence". En J.H. Ausubel y R. Herman (eds.): Cities and theirVital Systems Infrastructure: past, present, and future. NationalAcademy Press. Washington D.C.: 85-97.

BAILEY, G.N. (1983): "Concepts of time in Quaternary prehistory". Annual Review of Anthropology, 12: 165-92.

BAK, P. y CHEN, K. (1991): "Self-organized criticality". Scientific American , 264: 46-53.

BARRETT, J. (1987): "Fields of discourse: reconstituting a social archaeology". Critique ofAnthropology, 7: 5-16.

- (1992): Fragments from antiquity. Blackwell. Oxford.

Bender, J. y Wellbery, D.E. (eds.) (1991): Chronotypes: the construction of time. Stanford University Press. Stanford.

BENDER, B. (ed.) (1993): Landscape, politics and perspectives. Berg. Oxford.

Bergson, H. (1932): L'évolution créatrice. Félix Alcan. París.

Bintliff, J. (1991): The Annales School and Archaeology. Leicester University Press. Leicester.
BRAUDEL, F. (1953): El Mediterráneo y el mundo mediterráneo en la época de Felipe II. Fondo de Cultura Económica. México.

- (1991): Escritos sobre la historia. Alianza. Madrid.

ConRad, M. (1986): "What is the use of chaos?". En A. Holden (ed.): Chaos. Manchester University Press. Manchester: 3-14.

Dawkins, R. (1986): The Blind Watchmaker. Longman. Londres.

DurkheIm, E. (1915): Elementary Forms of the Religious Life. Unwin. Londres.

FEdorofF, N. y CourTy, M-A. (1995): "Le role respectif des facteurs anthropiques et naturels dans la dynamique actuelle et passée des paysages méditerranéens: cas du bassin de Vera, sud-est de l'Espagne". En S.E. van der Leeuw (ed.): L'homme et la degradation de l'environnement, XVe Rencontres Internationales d'Archéologie et d'histoire d'Antibes. Editions APDCA. Juan Les Pins: $134-148$.

FISCHER, D.H. (1976): "The braided narrative: substance and form in social history". En A. Fletcher (ed.): The Literature of Fact. Academic Press. New York: 109. 134.

FRASER, J.T. (1981): The Voices of Time. Amherst. Mass.

- (1987): Time: the familiar stranger. Amherst. Mass.

FuKuYAMA, F. (1992): El fin de la historia y el último hombre. Planeta. Barcelona.

Giddens, A. (1986): The Constitution of Society. Polity Press. Cambridge.

González Marcén, P. y Picazo, M. (1998): El Tiempo en Arqueología. Arco Libros. Madrid.

Gosden, C. (1994): Social Being and Time. Blackwell. Oxford.

Hahn, H-C. (1976): "Hora". En C. Brown (ed.): The New International Dictionary of New Testament Theology, 3. Paternoster Press. Exeter: 845-849.

HAYles, N.K. (1991): Chaos and Order: Complex Dynamics in Literature and Science. Chicago University Press. Chicago.

HODDER, I. (1987): “The contribution of the long term.”. En I. Hodder (ed.): Archaeology as Long Term History. Cambridge University Press. Cambridge: 1-8.

- (1988): Interpretación en Arqueología. Crítica. Barcelona.

- (1992): Theory and practice in archaeology. Routledge. Londres.

Holling, C.S. (1986): "The resilience of terrestrial ecosystems: local surprise and global change". En W.C. Clark y R.E. Munn (eds.): Sustainable development of the biosphere. Cambridge University Press. Cambridge: 292-320.

HubERMAN, B. (1988): The écology of computation. North Holland. Amsterdam.

JAMESON, F. (1991): El Posmodernismo o la lógica cultural del capitalismo avanzado. Paidós. Barcelona. 
Knapp, A.B. (1992): Archaeology, Annales, and Ethnohistory. Cambridge University Press. Cambridge.

Kracauer, S. (1966) "Time and history". History and Theory, 6: 65-78.

LECCARDI, C. (1996): "Rethinking social time: feminist perspectives". Time and Society, 5(2): 169-186.

LoRENZ, E. (1963): "Deterministic non periodic flow". Journal of Atmosferic Science 20: 130-141.

McGlade, J. (1987): "Chronos and the oracle: some thoughts on time, time-scales and simulation".Archaeological Review from Cambridge: 27-36.

- (1995a): "Archaeology and the ecodynamics of humanmodified landscapes". Antiquity , 69: 113-132.

- (1995b): "An integrative multiscalar modelling framework for human ecodynamic research in the Vera basin, south-east Spain". En S.E. van der Leeuw (ed.): L'homme et la dégradation de l'environnement. $\mathrm{XV}^{\mathrm{e}}$ Rencontres Internationales d'Archéologie et d'histoire d'Antibes. Editions APDCA. Juan-Les-Pins: 357-385.

- (1997a): "The limits of social control: coherence and chaos in a prestige-goods economy". En S.E. van der Leeuw y J. McGlade (eds.): Archaeology, time and structured transformation. Routledge. London: 298330.

- (1997b): "Estabilitat i inestabilitat en demografia prehistórica: una perspectiva de modelizació dinàmica". Cota Zero, 13: 103-116.

- (1999): "Archaeology and the evolution of cultural landscapes: towards an interdisciplinary research agenda”. En P.J. Ucko y R. Layton (eds.): Anthropological and ArchaeologicalApproaches to the Cultural Landscape. Routledge. Londres: 458-482.

MacGlade, J. y VAN DER LeEuw, S.E. (1997): “Archaeology and nonlinear dynamics: new approaches to long-term change". En S.E. van der Leeuw y J. McGlade (eds.): Time, process and structured transformation in archaeology. Routledge. Londres: 1-31.

Nicolis, G. y I. PRIGOGINE (1977): Self-Organization in Nonequilibrium Systems. John Wiley and Sons. New York.

Picazo, M. (1996): "Hearth and Home: the timing of maintenance activities". En J. Moore y E. Scott (eds.): Invisible People and Processes: writing gender and childohood in to EuropeanArchaeology. Leicester University Press. Londres: 59-67.

Prigogine, I. y Stengers, I. (1979): La Nouvelle Aliance. Galimard. Paris.
Ruelle, D. y F. TAKENS (1971): "On the nature of turbulence". Communications of Mathematical Physics , 20: 167-192.

SARACENo, C. (1993): "Continuita e discontinuita nella riproduzione sociale". En A. Carbonaro y C. Facchini (eds.): Biografie e costruzione dell'identita. Angeli. Milan: 37-55.

Schutz, A. (1962): The Problem of Social Reality. Martinus Nijhoff. La Haya.

Shanks, M. y Tilley, C. (1987): Social Theory and Archaeology. Cambridge University Press. Cambridge.

SHEMER, M. (1995): "Exorcising Laplace's demon: chaos and antichaos, history and metahistory". History and Theory, 34(1): 59-81.

Simiand, F. (1903): "Méthode historique et science sociale". Review, 9 (1985-86): 163-213.

Sorokin, P.A. y Merton, R.K. (1937): "Social time: a methodological and functional analysis". American Journal of Sociology, 42: 615-629.

STARr, C.G. (1966): "Historical and philosophical time". History and Theory, 6: 24-35.

Stewart, I. (1991): ¿Juega Dios a los dados? La nueva matemática del caos. Crítica. Barcelona.

Thomas, J. (1991): Rethinking the Neolithic. Cambridge University Press. Cambridge.

- (1993): "The hermeneutics of megalithic space". En C. Tilley (ed.): Interpretative Archaeology. Berg. Oxford.

Tilley, C. (ed.) (1993): Interpretative Archaeology. Berg. Oxford.

VAN DER LEEUW, S.E. (1990): "Rythmes temporels, espaces naturels et espaces vécus". En J.L. Fiches y S.E. van der Leeuw (eds.): Archéologie et Espaces. $\mathrm{X}^{\mathrm{e}}$ Rencontres Internationales d'Archéologie et d' histoire d'Antibes. Editions APDCA. Juan-Les-Pins: 299-346.

VAN DER Leeuw, S.E. y McGlade, J. (1993): "Information, cohérence et dynamiques urbaines". En D. Pumain y B. Lepetit (eds.): Temporalités Urbaines. Economica. Paris: 195-245.

- (1997a): Archaeology: time and structured transformation. Routledge. Londres.

- (1997b): "Structural change and bifurcation in urban evolution: a nonlinear dynamical perspective". En: S.E. van der Leeuw y J. McGlade (eds.): Archaeology: time and structured transformation. Routledge. Londres: 331-372.

White, H. (1971): Metahistory. Johns Hopkins University Press. Baltimore.

T. P., 56, n. ${ }^{\circ} 2,1999$ 